\title{
Participation of the microbiome in the pathogenesis of diabetes mellitus
}

\section{ABSTRACT}

Recent research works suggest that an altered, quantitative composition and qualitative diversity of the gut microbiome may play an important role in the development of metabolic disorders. Growing evidence suggests that the gut bacteria contribute to the onset of the low-grade inflammation, characterising also metabolic disorders arising via mechanisms of the gut barrier dysfunction. The gut microbiome composition has been associated with several components of metabolic syndrome, obesity, type 2 diabetes, chronic cardiovascular diseases, and non-alcoholic liver steatosis. The composition of the gut microbiome also represents an important, environmental factor that influences the development of type 1 diabetes. Many studies have examined the mechanisms by which the control of the microbiomes may play a role in the prevention and treatment of type 1 diabetes. (Clin Diabetol 2017; 6, 5: 178-181)

Key words: gut microbiome, pathogenesis of obesity, insulin resistance, type 2 diabetes, type 1 diabetes

\section{Introduction}

The term 'microbiome' refers to the aggregate of microorganisms which reside in the host. It has been established that the number of the microbiome cells is

Address for correspondence:

lek. Artur Chwalba

Medical Specialist Centre in Gliwice

e-mail: artur.adam.chwalba@gmail.com

Clinical Diabetology 2017, 6, 5, 178-181

DOI: $10.5603 /$ DK.2017.0029

Received: 04.05.2017

Accepted: 08.10.2017
10 times higher than the number of cells that form an organism of a healthy human, and its weight reaches approx. $2 \mathrm{~kg}$. It has multiple influences onto the human organism, hence recent years have seen the intensification of experimental as well as clinical studies aiming at the determination of the range and mechanism of these reactions.

Considerable progress is observed particularly in studies devoted to the composition of the gut microbiome and its effect on the incidence rate of different illness. In recent years, several associations between common chronic human disorders and altered gut microbiome composition and function have been reported [1, 2].

The gut microbiota have been considered a superorgan, which has coevolved with the host organism. Gut microbiota not only provide energy for the host, but also can shape mucosal and systemic immunity through their metabolites or bacterial peptides. Both animal models and human studies suggests that altered gut microbiota composition could play a causative role in the development of diabetes type 1, type 2 and obesity. Tai et al. presented a comprehensive discussion about the role of gut microbiota in the development of type 1, type 2 diabetes and obesity [3].

The intestinal microbiota has been well-known for a long time, but due to its newly recognised functions, clinicians' attention has turned to it again over the last decade. About 100.000 billion bacteria are present in the human intestines. Normal bacteriota defends the organism against the penetration of harmful microorganisms, and has many other functions in the gut wall integrity, innate immunity, insulin sensitivity, metabolism, and it is in cross-talk with the brain [4].

Intestinal bacteria play a crucial role in maintaining systemic and intestinal immune and metabolic homeostasis because of their effect on nutrient absorption and immune development and function [5]. 
Pathophysiological role of the gut microbiota in the development of obesity and insulin resistance

In experimental studies the importance of Toll-like receptors (TLRs) in regulating insulin sensitivity was found [6]. The aim of the mentioned study was to investigate the effect of the microbiota on metabolic parameters, glucose tolerance, insulin sensitivity and signalling in TLR2 deficient mice. Similar observations have been carried out by other authors [7, 8]. Differences in the microbiota composition, functional genes, and metabolic activities are observed between obese and lean individuals, suggesting a contribution of the gut microbiota to these phenotypes. Obesity is often accompanied by dyslipidaemia, hypertension, and impaired glucose homeostasis, known as the metabolic syndrome. In the last decade the intestinal microbiota has been proposed as another environmental factor involved in the onset of obesity. Overweight and obesity increase the risk for a number of diseases, namely, cardiovascular diseases, type 2 diabetes, dyslipidaemia, premature death, non-alcoholic fatty liver disease, as well as different types of cancer [9]. Data are accumulating in animal models and humans suggesting that obesity is associated with a profound dysbiosis [10]. Alteration of the microbiota composition may stimulate the development of obesity and other metabolic diseases. Gut microbiota exerts a significant role in the pathogenesis of the metabolic syndrome [11]. The composition of the gut microbiome can have its effect on the occurrence of insulin resistance, as well as obesity, in different ways. Recently, this issue has been presented by Saad et al. [12]. These authors pointed out that these studies should open new therapeutic avenues for obesity and insulin resistance and its comorbidities. Phenomena which can be mentioned among the mechanisms by which the gut microbiota could contribute to obesity and metabolic diseases, are - inter alia - increased intestinal permeability for bacterial lipopolysaccharides (LPS) in response to the consumption of high-fat diets resulting in an elevated systemic LPS level and low-grade inflammation [13].

Another mechanism is the increase of the density of capillary walls in the epithelium of the small intestine due to the presence of the pathological microbiome. The mechanisms referred to above increase the volume and rate of absorption of monosaccharides from the intestine.

The gut microbiota plays a crucial role in gastrointestinal mucosa permeability and regulates the fermentation and absorption of dietary polysaccharides, which may explain its importance in the regulation of fat accumulation and the resultant development of obesity-related diseases [14].

\section{Obesity and metabolic diseases and their link to the intestinal microbiota}

The gut bacteria exert beneficial and harmful effects in metabolic diseases. The research that is carried out in this respect comprises studies on both animal models and humans, designed to determine the differences in the composition of the intestinal microbiota and its role in various disease states [15-17].

An altered composition of the microbiome may also foster the increase of the absorption volume of shortchain fatty acids from the intestine to the host's blood. Their increased level stimulates the secretion of the $Y Y$ peptide in the fatty acid. The intestine motility slows down then, which favours the increase in the proportions of nutrients absorption compared to individuals with the physiological microbiome. Disorders of the type predispose to the occurrence of obesity $[13,15,17]$.

Studies of Xiao et al. have shown that chronic inflammation induced by endotoxins from a dysbiotic gut microbiota contributes to the development of obesityrelated metabolic disorders. Modification of the gut microbiota by a diet to balance its composition becomes a promising strategy to help manage obesity [18]

\section{Diabetes and the microbiome}

In 2014 a research symposium titled 'Diabetes and the Microbiome', sponsored by the American Diabetes Association and JDRF, was held in Chicago. The agenda of the symposium covered a discussion devoted to findings of combining the pathophysiology of diabetes with the microbiome and the potential opportunities to apply these studies in prevention and treatment practices. During the debate the agenda of the future multicentre trial devoted to the microbiome and its role in the pathogenesis of type 1 and type 2 diabetes was planned, as well as experiments on animal models and clinical trials on humans [19].

A dramatic increase in the incidence of diabetes, observed over the past 50 years, more often links the gut microbiome composition and function to antipancreatic immunity, insulin-resistance, and obesity investigations, as indicated by studies both in rodent models and in human studies [20]. The agenda of these studies covers multiple directions of the pathogenesis of such metabolic disorders as changes in the immunological reactions contributing to the incidence of diabetes, as well as pathogenetic significance of inflammations in these diseases [12, 21-25].

In the study devoted to the intestinal microflora in mice a positive relation between the liposaccharide (LPS) content in the blood plasma on one hand and weight gain, triglyceride accumulation, insulin resistance and type 2 diabetes on the other was observed. 
LPS is a proinflammatory element of the cell wall of bacteria that form the intestinal microflora and it can contribute to the development of inflammation that accompanies type 2 diabetes [26].

Intravenous administration of this lipopolysaccharide to mice causes insulin resistance and obesity. The results obtained in experimental trials have been confirmed in trials performed in human subjects. An elevated LPS level has been detected in patients suffering from diabetes compared to healthy subjects. This might be suggestive of the participation of LPS in the pathogenesis of type 2 diabetes. Studies targeted at the establishment of the possibility of modulation of the gut flora by means of probiotics are being undertaken, as well.

It is important to establish the pathogenic relation between the gut microbiota and diabetes since it might lead to the development of integrated strategies using probiotics to prevent and treat these metabolic disorders [21]. During the last decade, evidence has accumulated to support a role for gut microbiota in glycaemic control and type 2 diabetes. The Swedish authors have recently presented review the results of research on composition and functionality of human gut microbiota in type 2 diabetes [27].

\section{Role of microbiota in the pathogenesis of type 1 diabetes (T1D)}

Type 1 diabetes is an autoimmune disorder characterised by the selective destruction of insulin-producing $\beta$ cells as a result of a complex interplay between genetic and environmental factors in genetically susceptible individuals. An increasing amount of experimental data from animal models and humans has supported the role played by imbalanced gut microbiome in T1D [28-30].

He et al. based on the literature from the years 2004 to 2014 presented the results of research into the effects of the gut microbiota on diabetes, energy metabolism, inflammation, the immune system, gut permeability, and insulin resistance [31]. These observations may have implications for the design of new microbiota-based therapies in the prevention and treatment of T1D [32].

Studies that arouse considerable interest are the ones that examine relations between the incidence of the type 1 diabetes and the type of bacteria that inhabit intestines. In the type 1 diabetes an altered composition of the gut microbiota compared to the microbiota in healthy subjects has been observed. A phenomenon that is listed as one of the causes of the increase in the incidence rate of autoimmune diseases, type 1 diabetes included, observed particularly in such countries as e.g. Finland, is the 'hygiene hypothesis'. Studies conducted among infants and young children in Finland and Estonia have demonstrated that in this area the dominating species of the gut bacteria are the ones that activate the immunological system particularly strongly.

Very broad observations pertaining to differences in the composition of the bacterial microbiome and the incidence of early-onset autoimmune diseases which are common in Finland and Estonia, but less prevalent in Russia, have been presented by Vatanen et al. [25]. They have pointed to the fact that changes in the bacterial microflora can be detected even before any clinical symptoms of the type 1 diabetes are observable [33].

The role of Thelper type 17 (Th17) cells along with impaired T regulatory (Treg) cells in the development of T1D in animal models and humans is taken into consideration. Alteration of the gut microbiota has been implicated to be responsible for the imbalance between Th17 and Treg cells [34].

There are attempts to explain the protective role of Th17 cells in the targeted therapy in children with islet autoimmunity, and the possibilities of their use in the prevention of progression to overt diabetes.

Experimental research carried out by Peng et al. has shown that altering the gut bacteria can have a lasting effect on alteration of the gut microbiome and the gut mucosal immunity, and it can ameliorate autoimmunity [35]. By altering the bacterial composition, the interactions of these gut bacteria with the mucosal immune system can induce regulatory CD8 T cells that may influence the development of autoimmune diabetes.

Recently, italian authors presented results of comparative studies the inflammatory status and microbiome composition evaluated in biopsies of the duodenal mucosa of patients with type 1 diabetes compared to patients with celiac disease and healthy controls. This study shows that duodenal mucosa in T1D presents disease specific abnormalities in inflammatory profile and microbiota.

This is important for understanding the mechanisms underlying the complex pathogenesis of T1D and indicate new perspectives for future therapies targeting the intestine [36].

\section{Conclusions}

A dramatic increase in the prevalence of obesity, metabolic syndrome, and diabetes in all age groups, observed in recent years, tends to intensify the search for mechanisms leading to these disorders. The previous genetic studies are known to demonstrate a huge role played by environmental factors. Recently, more and more interest is focused on the composition and function of the gut microbiome. 


\section{REFERENCES}

1. Shreiner AB, Kao JY, Young VB. The gut microbiome in health and in disease. Curr Opin Gastroenterol. 2015; 31(1): 69-75, doi: 10.1097/MOG.0000000000000139, indexed in Pubmed: 25394236.

2. Cho I, Blaser MJ. The human microbiome: at the interface of health and disease. Nat Rev Genet. 2012; 13(4): 260-270, doi: 10.1038/nrg3182, indexed in Pubmed: 22411464.

3. Tai N, Wong FS, Wen Li. The role of gut microbiota in the development of type 1, type 2 diabetes mellitus and obesity. Rev Endocr Metab Disord. 2015; 16(1): 55-65, doi: 10.1007/s11154-0159309-0, indexed in Pubmed: 25619480.

4. Halmos T, Suba I. [Physiological patterns of intestinal microbiota. The role of dysbacteriosis in obesity, insulin resistance, diabetes and metabolic syndrome]. Orv Hetil. 2016; 157(1): 13-22, doi: 10.1556/650.2015.30296, indexed in Pubmed: 26708682.

5. Needell JC, Zipris D. The Role of the Intestinal Microbiome in Type 1 Diabetes Pathogenesis. Curr Diab Rep. 2016; 16(10): 89, doi: 10.1007/s11892-016-0781-z, indexed in Pubmed: 27523648.

6. Caricilli AM, Picardi PK, de Abreu LL, et al. Retraction: Gut Microbiota Is a Key Modulator of Insulin Resistance in TLR 2 Knockout Mice. PLoS Biol. 2016; 14(5): e1002479, doi: 10.1371/journal. pbio.1002479, indexed in Pubmed: 27213533.

7. Ehses JA, Meier DT, Wueest S, et al. Toll-like receptor 2-deficient mice are protected from insulin resistance and beta cell dysfunction induced by a high-fat diet. Diabetologia. 2010; 53(8): 1795-1806, doi: 10.1007/s00125-010-1747-3, indexed in Pubmed: 20407745.

8. Nackiewicz D, Dan M, He W, et al. TLR2/6 and TLR4-activated macrophages contribute to islet inflammation and impair beta cell insulin gene expression via IL-1 and IL-6. Diabetologia. 2014; 57(8): 1645-1654, doi: 10.1007/s00125-014-3249-1, indexed in Pubmed: 24816367.

9. Kobyliak N, Virchenko O, Falalyeyeva T. Pathophysiological role of host microbiota in the development of obesity. Nutr J. 2016; 15: 43, doi: 10.1186/s12937-016-0166-9, indexed in Pubmed: 27105827.

10. Tilg $H$, Moschen AR. Microbiota and diabetes: an evolving relationship. Gut. 2014; 63(9): 1513-1521, doi: 10.1136/ /gutjnl-2014-306928, indexed in Pubmed: 24833634.

11. Festi D, Schiumerini R, Eusebi $L$, et al. Gut microbiota and metabolic syndrome. World J Gastroenterol. 2014; 20(43): 16079-16094, doi: 10.3748/wjg.v20.i43.16079, indexed in Pubmed: 25473159.

12. Saad MJA, Santos A, Prada PO. Linking Gut Microbiota and Inflammation to Obesity and Insulin Resistance. Physiology (Bethesda). 2016; 31(4): 283-293, doi: 10.1152/physiol.00041.2015, indexed in Pubmed: 27252163.

13. Blaut M. Gut microbiota and energy balance: role in obesity. Proc Nutr Soc. 2015; 74(3): 227-234, doi: 10.1017/ /S0029665114001700, indexed in Pubmed: 25518735.

14. Lau E, Carvalho D, Pina-Vaz C, et al. Beyond gut microbiota: understanding obesity and type 2 diabetes. Hormones (Athens). 2015; 14(3): 358-369, doi: 10.14310/horm.2002.1571, indexed in Pubmed: 26188221.

15. Gérard P. Gut microbiota and obesity. Cell Mol Life Sci. 2016; 73(1): 147-162, doi: 10.1007/s00018-015-2061-5, indexed in Pubmed: 26459447

16. Tremaroli V, Bäckhed F. Functional interactions between the gut microbiota and host metabolism. Nature. 2012; 489(7415): 242-249, doi: 10.1038/nature11552, indexed in Pubmed: 22972297.

17. Woting A, Blaut M. The Intestinal Microbiota in Metabolic Disease. Nutrients. 2016; 8(4): 202, doi: 10.3390/nu8040202, indexed in Pubmed: 27058556.

18. Xiao $\mathrm{S}$, Fei $\mathrm{Na}$, Pang $\mathrm{X}$, et al. A gut microbiota-targeted dietary intervention for amelioration of chronic inflammation underlying metabolic syndrome. FEMS Microbiol Ecol. 2014; 87(2): 357-367, doi: 10.1111/1574-6941.12228, indexed in Pubmed: 24117923.

19. Semenkovich CF, Danska J, Darsow T, et al. American Diabetes Association and JDRF Research Symposium: Diabetes and the Microbiome. Diabetes. 2015; 64(12): 3967-3977, doi: 10.2337/ /db15-0597, indexed in Pubmed: 26420863.

20. Paun A, Danska JS. Modulation of type 1 and type 2 diabetes risk by the intestinal microbiome. Pediatr Diabetes. 2016; 17(7): 469-477, doi: 10.1111/pedi.12424, indexed in Pubmed: 27484959.

21. Delzenne NM, Cani PD, Everard A, et al. Gut microorganisms as promising targets for the management of type 2 diabetes. Diabetologia. 2015; 58(10): 2206-2217, doi: 10.1007/s00125015-3712-7, indexed in Pubmed: 26224102.

22. Blandino G, Inturri R, Lazzara F, et al. Impact of gut microbiota on diabetes mellitus. Diabetes Metab. 2016; 42(5): 303-315, doi: 10.1016/j.diabet.2016.04.004, indexed in Pubmed: 27179626.

23. Everard A, Cani PD. Diabetes, obesity and gut microbiota. Best Pract Res Clin Gastroenterol. 2013; 27(1): 73-83, doi: 10.1016/j. bpg.2013.03.007, indexed in Pubmed: 23768554.

24. Remely M, Aumueller E, Jahn D, et al. Microbiota and epigenetic regulation of inflammatory mediators in type 2 diabetes and obesity. Benef Microbes. 2014; 5(1): 33-43, doi: 10.3920/ /BM2013.006, indexed in Pubmed: 24533976.

25. Vatanen T, Kostic AD, d'Hennezel E, et al. DIABIMMUNE Study Group. Variation in Microbiome LPS Immunogenicity Contributes to Autoimmunity in Humans. Cell. 2016; 165(4): 842-853, doi: 10.1016/j.cell.2016.04.007, indexed in Pubmed: 27133167.

26. Wang F, Zhang C, Zeng Q. Gut microbiota and immunopathogenesis of diabetes mellitus type 1 and 2. Front Biosci. 2016; 21(5): 900-906, doi: 10.2741/4427.

27. Brunkwall L, Orho-Melander M. The gut microbiome as a target for prevention and treatment of hyperglycaemia in type 2 diabetes: from current human evidence to future possibilities. Diabetologia. 2017; 60(6): 943-951, doi: 10.1007/s00125-0174278-3, indexed in Pubmed: 28434033.

28. Gianchecchi E, Fierabracci A. On the pathogenesis of insulindependent diabetes mellitus: the role of microbiota. Immunol Res. 2017; 65(1): 242-256, doi: 10.1007/s12026-016-8832-8, indexed in Pubmed: 27421719.

29. Livanos $A E$, Greiner TU, Vangay $P$, et al. Antibiotic-mediated gut microbiome perturbation accelerates development of type 1 diabetes in mice. Nat Microbiol. 2016; 1(11): 16140, doi: 10.1038/ /nmicrobiol.2016.140, indexed in Pubmed: 27782139

30. Paun A, Yau C, Danska JS, et al. Immune recognition and response to the intestinal microbiome in type 1 diabetes. J Autoimmun. 2016; 71(2): 10-18, doi: 10.1016/j.jaut.2016.02.004, indexed in Pubmed: 26908163.

31. He C, Shan $Y$, Song W. Targeting gut microbiota as a possible therapy for diabetes. Nutr Res. 2015; 35(5): 361-367, doi: 10.1016/j.nutres.2015.03.002, indexed in Pubmed: 25818484.

32. Gülden E, Wong FS, Wen Li. The gut microbiota and Type 1 Diabetes. Clin Immunol. 2015; 159(2): 143-153, doi: 10.1016/j. clim.2015.05.013, indexed in Pubmed: 26051037.

33. Dunne JL, Triplett EW, Gevers D, et al. The intestinal microbiome in type 1 diabetes. Clin Exp Immunol. 2014; 177(1): 30-37, doi: 10.1111/cei.12321, indexed in Pubmed: 24628412.

34. Li Y, Liu Yu, Chu CQ. Th17 Cells in Type 1 Diabetes: Role in the Pathogenesis and Regulation by Gut Microbiome. Mediators Inflamm. 2015; 2015: 638470, doi: 10.1155/2015/638470, indexed in Pubmed: 26843788.

35. Peng J, Narasimhan S, Marchesi JR, et al. Long term effect of gut microbiota transfer on diabetes development. J Autoimmun. 2014; 53: 85-94, doi: 10.1016/j.jaut.2014.03.005, indexed in Pubmed: 24767831.

36. Pellegrini S, Sordi V, Bolla AM, et al. Duodenal Mucosa of Patients With Type 1 Diabetes Shows Distinctive Inflammatory Profile and Microbiota. J Clin Endocrinol Metab. 2017; 102(5): 1468-1477, doi: 10.1210/jc.2016-3222, indexed in Pubmed: 28324102. 